\title{
Tumor Size at Magnetic Resonance Imaging Association With Lymph Node Metastasis and Lymphovascular Space Invasion in Resectable Cervical Cancer A Multicenter Evaluation of Surgical Specimens
}

\author{
Xiao-li Chen, MS, * Guang-wen Chen, MS, $\dagger$ Guo-hui Xu, MS, * Jing Ren, MS, * \\ Zhen-lin Li, MS, $\neq$ Hong Pu, MS, $†$ and Hang $\mathrm{Li}, \mathrm{MS} \dagger$
}

\begin{abstract}
Objective: To determine whether gross tumor volume (GTV) and the maximum diameter of resectable cervical cancer at magnetic resonance imaging (MRI) could predict lymph node metastasis (LNM) and lymphovascular space invasion (LVSI).

Materials and Methods: A total of 315 consecutive patients with cervical cancer were retrospectively identified. Gross tumor volume and the maximum diameter of tumor were evaluated on MRI. Univariate and multivariate logistic regression analyses were performed to determine whether tumor size could predict LNM and LVSI. Cutoffs of GTV, maximum diameter, and the International Federation of Gynecology and Obstetrics (FIGO) classification of tumor were first investigated in 255 patients (group A) and then validated in an independent cohort of 60 patients (group B) using area under the receiver operating characteristic curve (AUC) analysis for predicting the presence of LNM and LVSI.

Results: Univariate analysis showed that GTV and the maximum diameter of tumor could predict LNM and LVSI (all $P<0.0001$ ). Multivariate analyses indicated GTV as an independent risk factor of LNM and LVSI (all $P<0.0001$ ). In group A, GTV, the maximum diameter, and the FIGO stage could identify LNM (AUC, 0.813, 0.741, and 0.69, respectively) and LVSI (AUC, 0.806, 0.751, and 0.684, respectively). In group B, GTV, the maximum diameter, and the FIGO stage could help identify LNM (AUC, 0.902, 0.825, and 0.759 , respectively) and LVSI (AUC, 0.771, 0.748, and 0.700 , respectively).

Conclusions: Gross tumor volume and the maximum diameter of resectable cervical cancer at MRI demonstrated capability in predicting LNM and LVSI, which were more accurate than FIGO stage.
\end{abstract}

Key Words: MRI, Tumor size, Cervical cancer, Lymph node metastasis, Lymphovascular space invasion

*Department of Radiology, Sichuan Cancer Hospital and Institute, Sichuan Cancer Center, School of Medicine, University of Electronic Science and Technology of China, Chengdu, China; $\uparrow$ Department of Radiology, Affiliated Hospital of Medical School, University of Electronic Science and Technology of China, Sichuan Academy of Medical Sciences and Sichuan Provincial People’s Hospital; and \$Department

Copyright (C) 2018 The Author(s). Published by Wolters Kluwer Health, Inc. on behalf of IGCS and ESGO. This is an openaccess article distributed under the terms of the Creative Commons Attribution-Non Commercial-No Derivatives License 4.0 (CCBYNC-ND), where it is permissible to download and share the work provided it is properly cited. The work cannot be changed in any way or used commercially without permission from the journal. ISSN: $1048-891 \mathrm{X}$

DOI: $10.1097 /$ IGC.0000000000001327 of Radiology, West China Hospital of Sichuan University, Chengdu, Sichuan, China.

Address correspondence and reprint requests to Hang Li, MS, Department of Radiology, Sichuan Academy of Medical Sciences and Sichuan Provincial People's Hospital, 32\# Second Section of First Ring Road, Qingyang District, Chengdu, Sichuan 610070, China. E-mail: lihang111222@126.com; Xiao-li Chen, MS, Department of Radiology, Sichuan Cancer Hospital and Institute, Sichuan Cancer Center, School of Medicine, University of Electronic Science and Technology of China, Chengdu 610041, China. E-mail: 20shenbaise@163.com.

This study was supported by the Project of Huimin Technology of Chengdu (No. 2015-HM01-00082-SF and No. 2015-HM01-00164-SF).

The authors declare no conflicts of interest or industry supports of the project in this study.

Guang-wen Chen, Hong Pu, and Guo-hui Xu contributed equally to this study. 
Received February 17, 2018, and in revised form May 22, 2018.

Accepted for publication June 4, 2018.

(Int J Gynecol Cancer 2018;28: 1545-1552)

C ervical cancer is one of the most common diagnosed cancers and one of the leading causes of cancer death in females worldwide, especially in developing countries. ${ }^{1}$ The treatment of cervical cancer involves surgery and chemoradiotherapy, which depends on early detection and accurate staging, especially the lymph node involvement. ${ }^{2-4}$ Lymph node metastasis (LNM) is not only closely related to cervical cancer recurrence and distant metastasis, but also an independent factor in poor survival. Furthermore, lymphovascular space invasion (LVSI) not only was an independent influencing factor for LNM but also has been well considered for treatment cervical cancer now. ${ }^{5}$ Lymph node metastasis and LVSI were important poor prognostic factors for survival regardless of the disease extent. 6,7 The accurate diagnosis of LNM and LVSI is currently considered an important prognostic factor for survival and making the choice of individualized treatment to the cervical cancer patients.

Conventional radiology is still extremely useful in diagnosing cervical cancer. The greatest restriction of using ultrasound is the low sensitivity in detecting LNM. ${ }^{8,9}$ Magnetic resonance imaging (MRI) is a safe, repeatable, and effective imaging technique that has played a progressively important role in identifying the cervical tumor and its parametrial invasion. ${ }^{10}$ However, preoperatively detecting LNM has been a major drawback with a wide range sensitivity of $29 \%$ to $86 \%$ because it may be difficult to identify micrometastases lymph nodes that have normal size. ${ }^{11-13}$ Even if it used the expensive positron emission tomography/computed tomography for assessing regional LNM, the diagnostic accuracy is low in early-stage disease. ${ }^{14}$ After pathological correlations, the authors found that the positron emission tomography/ computed tomography was falsely negative in $12 \%$ of 132 patients, especially for lymph nodes with $5 \mathrm{~mm}$ or less diameter. ${ }^{15}$ Previous studies reported that the gross tumor volume (GTV) of cervical cancer on MRI could be used for predicting the presence of histologic LNM and treatment outcome. ${ }^{16}$ It has been reported that the tumor volume and LVSI were all critical for selecting an appropriate therapeutic modality. ${ }^{17}$ The previously reported study also demonstrated that the tumor size of cervical cancer on MRI was important for preoperative staging and influenced management decisions. ${ }^{5,18}$ Therefore, our study was to retrospectively assess whether the tumor size of resectable cervical cancer on MRI could predict regional LNM and LVSI.

\section{MATERIALS AND METHODS}

\section{Patients}

The institutional ethics committee approved this study. Between December 2015 and June 2017, we retrospectively analyzed the data of patients with cervical cancer treated at
3 hospitals. Patients who met the following criteria were included: (1) had histologically confirmed cervical cancer and received radical hysterectomy with lymphadenectomy, (2) underwent dynamic MRI within 1 week before surgery, and (3) without preoperative chemotherapy or radiotherapy. Consequently, this study involved 315 patients. Then these patients were divided into 2 cohorts. In the development cohort (group A), data in 255 patients were used to develop the GTV cutoff values in identifying LNM and LVSI. In the validation cohort (group B), data in 60 patients were used to validate the developed cutoff values of tumor size. The average age was 48 years (range, 29-74 years) in group A. The average age was 48.5 years (range, 31-70 years) in group B.

\section{Magnetic Resonance Imaging}

The patients undertook the examinations with standard body coil on 3.0-T Magnetom (Siemens, Munich, Germany) scanners. Imaging included sagittal 5-mm fast-spin echo T2-weighted images (echo time [TE], 90 milliseconds; repetition time [TR], 4600 milliseconds; number of excitations 2), and axial 5-mm T2-weighted and T1-weighted images (TE, 10 milliseconds; TR, 212 milliseconds; number of excitations, 2). The diffusionweighted imaging was performed in axial plane with $b$ values of $0,300,500$, and $800 \mathrm{~s} / \mathrm{mm}^{2}$ using the following parameters: TR, 4007 milliseconds; TE, 55 milliseconds; slice thickness, $5 \mathrm{~mm}$; and field of view, $34 \mathrm{~cm}$. Gadolinium-enhanced fatsuppressed T1-weighted axial images and sagittal images were performed after approximately $20-\mathrm{mL}$ (a total of $0.2 \mathrm{mmol} / \mathrm{kg}$ of body weight) gadodiamide (gadopentetate dimeglumine; Consun, Guangzhou, China) injected via a pressure injector at a dosage of $2 \mathrm{~mL} / \mathrm{s}$ followed by a $20-\mathrm{mL}$ saline solution flush. It took approximately 30 minutes to complete all these MRI scans.

Tumor volume and the maximum diameter of tumor were evaluated quantitatively on the T2-weighted images. Two experienced radiologists who were blinded to each other's results and patient information independently analyzed the MR images. According to the previous reports, tumor volume was calculated by multiplying the sum of tumor areas by the section thickness. ${ }^{19,20}$ Tumor area was manually outlined on the sagittal or axial T2-weighted image images along the border of the each suspected tumor slice (Fig. 1). The true maximum diameter of tumor is the longest diameter measured on both sagittal and transversal MR images.

\section{Lymph Node Dissection and Histologic Evaluation}

The removed lymph nodes included the nodes around the external, internal, and common iliac vessels; in the obturator fossa; and in retroperitoneal space. The mean number of nodes resected during surgery across this patient population was 36 (range, 20-58). The primary tumor and nodes were sliced and marked with hematoxylin and eosin, and 


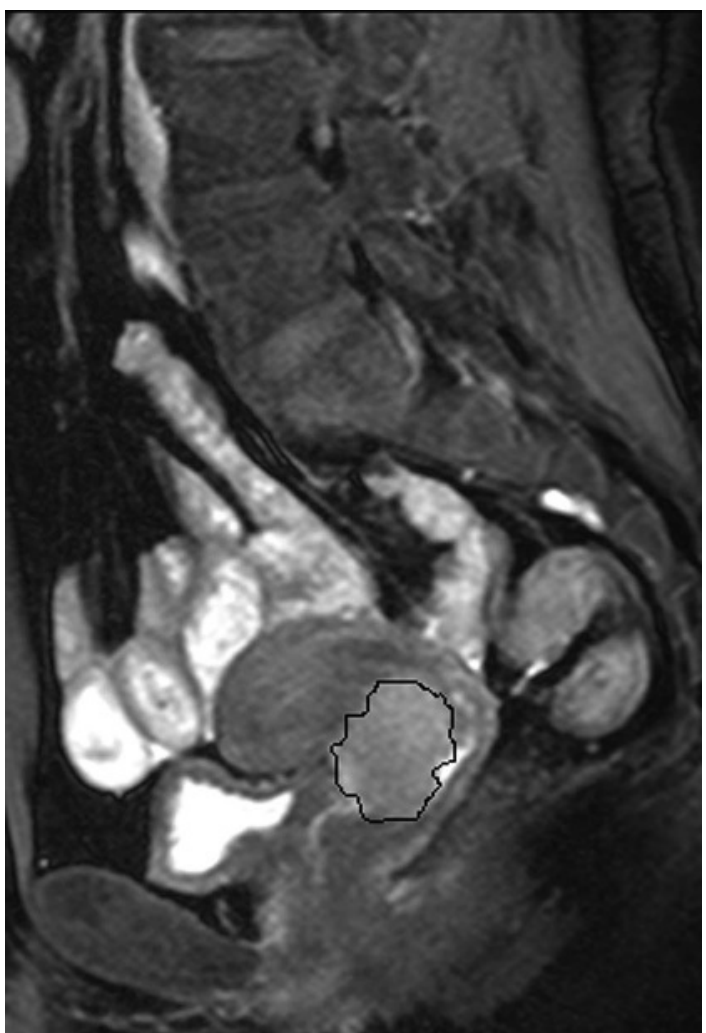

FIGURE 1. A 55-year-old woman with resectable cervical cancer. The tumor area is manually traced within the outline on the T2-weighted image sagittal MR image.

immunohistochemistry. The experienced pathologists microscopically observed the presence of LNM and LVSI.

\section{Statistical Analysis}

All statistical analyses were supported with SPSS (version 17.0; SPSS, Chicago, IL). Significant difference was considered if $P<0.05$. The MRI data were used to test interobserver reproducibility of the measurements. In these 315 patients, interobserver reproducibility of tumor size measurements was assessed using coefficient of variation (CV). Variability was considered slight when the $\% \mathrm{CV}$ was less than $10 \%$, and the final result was the average value of the 2 measurements. If the $\% \mathrm{CV}$ surpassed $10 \%$, the observers completed additional 2 measurements. Moreover, the final result was the average of the 4 measurements.

Univariate associations were analyzed using the $\chi^{2}$ test or Fisher exact test when applicable. Multivariate logistic regression analyses were used to assess the risk factors for LVSI and LNM. The area under the receiver operating characteristic (ROC) curve (AUC) was performed to assess the diagnostic efficiency of tumor size of resectable cervical cancer on MRI for predicting regional LNM and LVSI. The accuracy, sensitivity, specificity, positive predictive value, and negative predictive value were used for the identification of LNM and LVSI in resectable cervical cancer, with an optimum tumor size cutoff value that maximized the sum of sensitivity and specificity.

\section{RESULTS}

\section{Interobserver Variability of Measuring Tumor size}

For the first evaluation in this cohort, the mean GTV and the maximum diameter of tumor were $4.96 \pm 4.67 \mathrm{~cm}^{3}$ (range, $0.52-101.99 \mathrm{~cm}^{3}$ ) and $2.94 \pm 0.99 \mathrm{~cm}$ (range, $0.7-6.5 \mathrm{~cm}$ ), respectively. For the repeat measurement, the mean GTV and the maximum diameter of tumor were $5.18 \pm 4.71 \mathrm{~cm}^{3}$ (range, $0.67-111.6 \mathrm{~cm}^{3}$ ) and $2.91 \pm 0.99 \mathrm{~cm}$ (range, $0.6-6.5 \mathrm{~cm}$ ), respectively. As for the interobserver variability of the MRI measurements of GTV and the maximum diameter of tumor, the CVs were $8.42 \%$ (range, $2.3 \%-23.7 \%$ ) and $4.38 \%$ (range, $2.3 \%-10 \%$ ), respectively. Therefore, the interobserver variability of tumor size was small and average values of both measurements were regarded as the final value.

\section{Univariate and Multivariate Analyses of Clinicopathological Factors Correlated With LNM and LVSI}

The correlation between clinicopathological factors and LNM is shown in Table 1. The correlation between clinicopathological factors and LVSI is shown in Table 2. The distributions of GTV and the maximum diameter of tumor stratified by N stage and LVSI are shown in Figure 2. Histology type could not predict the LNM and LVSI. There was no difference between ages for predicting LVSI $(P=0.903)$. There was a significant difference between ages for predicting LNM $(P=0.005)$. Tumor differentiation, International Federation of Gynecology and Obstetrics (FIGO) category, the maximum diameter of tumor, and GTV could predict the LNM and LVSI (all $P<0.05$ ). Multivariate analysis showed GTV as an independent risk factor related to LNM $(P<0.001$; odds ratio, $1.286)$ and LVSI $(P<0.001$; odds ratio, 1.406).

\section{ROC Analyses of GTV and the Maximum Diameter of Resectable Cervical Cancer for Predicting the Presence of LNM and LVSI}

As illustrated in Table 3 and Figures 3A, B, in the development cohort, the maximum diameters of tumor cutoff value in identifying the presence of LNM and LVSI were 2.75 cm (AUC, 0.741; sensitivity, 80.2\%; specificity, 61.4\%; accuracy, $67.0 \%$ ) and $2.90 \mathrm{~cm}$ (AUC, 0.751 ; sensitivity, 68.4\%; specificity, $67.4 \%$; accuracy, $60.8 \%$ ), respectively. The cutoff values of GTV in identifying the presence of LNM and LVSI were $5.17 \mathrm{~cm}^{3}$ (AUC, 0.813 ; sensitivity, $75.6 \%$; specificity, $76.0 \%$; accuracy, $75.7 \%$ ) and $6.41 \mathrm{~cm}^{3}$ (AUC, 0.806 ; sensitivity, $60.2 \%$; specificity, $93.4 \%$; accuracy, $76.0 \%$ ), respectively. The figures show that the AUC value of FIGO for identifying the presence of LNM and LVSI is less than that of GTV and the maximum diameter. In the development cohort, the AUC values of FIGO for identifying the presence of LNM and LVSI were 0.69 and 0.684 , respectively.

As illustrated in Table 3 and Figures 3C, D in the validation cohort, when compared with data in the development cohort, the AUCs of GTV obtained in the validation cohort in the identification of LNM and LVSI were 0.902 and 0.771 , respectively. The AUCs of the maximum diameter 
TABLE 1. Univariate analysis of clinicopathological factors and gross tumor size of resectable cervical cancer correlated with regional LNM

\begin{tabular}{|c|c|c|c|}
\hline \multirow[b]{2}{*}{ Variables } & \multicolumn{2}{|c|}{ LNM } & \multirow[b]{2}{*}{$\boldsymbol{P}$} \\
\hline & $\begin{array}{l}\text { Negative } \\
(n=169)\end{array}$ & $\begin{array}{l}\text { Positive } \\
(n=86)\end{array}$ & \\
\hline Age, year & & & 0.005 \\
\hline$<50$ & $93(58.9)$ & $65(41.1)$ & \\
\hline$\geq 50$ & $76(78.4)$ & $21(21.6)$ & \\
\hline Histology type & & & 0.566 \\
\hline Adenocarcinoma & $14(66.8)$ & $9(33.2)$ & \\
\hline $\begin{array}{l}\text { Squamous cell } \\
\text { carcinoma }\end{array}$ & $155(60.9)$ & $77(39.1)$ & \\
\hline Tumor differentiation & & & 0.002 \\
\hline Poorly differentiated & $85(59.0)$ & $59(41.0)$ & \\
\hline Moderately differentiated & $36(67.9)$ & $17(32.1)$ & \\
\hline Highly differentiated & $48(82.8)$ & $10(17.2)$ & \\
\hline FIGO category & & & $<0.0001$ \\
\hline IB1 & $62(92.5)$ & $5(7.5)$ & \\
\hline IB2 & $41(60.3)$ & $27(39.7)$ & \\
\hline IIA1 & $36(64.3)$ & $20(35.7)$ & \\
\hline IIA2 & $10(45.5)$ & $12(54.5)$ & \\
\hline IIB & $20(47.6)$ & $22(52.4)$ & \\
\hline $\begin{array}{l}\text { Maximum diameter } \\
\text { of tumor, } \mathrm{cm}\end{array}$ & & & $<0.0001$ \\
\hline$<4$ & $154(69.1)$ & $69(30.9)$ & \\
\hline$\geq 4$ & $15(46.9)$ & $17(53.1)$ & \\
\hline GTV, $\mathrm{cm}^{3}$ & & & $<0.0001$ \\
\hline$<3.9$ & $108(85.0)$ & $19(15.0)$ & \\
\hline$\geq 3.9$ & $61(47.7)$ & $67(52.3)$ & \\
\hline LVSI & & & $<0.0001$ \\
\hline Absent & $133(75.1)$ & $44(24.9)$ & \\
\hline Present & $36(46.2)$ & $42(53.8)$ & \\
\hline
\end{tabular}

obtained in the validation cohort in the identification of LNM and LVSI were 0.825 and 0.748 , respectively. In Figures 3C, $\mathrm{D}$ also show that the AUC value of FIGO is less than that of GTV and the maximum diameter in identifying the presence of LNM and LVSI in the validation cohort. In the validation cohort, the AUC values of FIGO in identifying the presence of LNM and LVSI were 0.759 and 0.700 , respectively.

\section{DISCUSSION}

Lymph node metastasis and LVSI are not only closely related to cervical cancer recurrence and distant metastasis, but also independent factors for poor survival. ${ }^{5-7}$ Consequently, the accurate assessment of LNM and LVSI plays a significant role in prognosis assessment and treatment. Our results suggest that the tumor size measured on MRI could be a potential alternative way for preoperative distinguishing $\mathrm{N}$ stages and identifying LVSI of cervical cancer. The presence of LNM and LVSI is the most important risk factor that affects survival and prognosis, ${ }^{6,15}$ which are not involved in FIGO classification. ${ }^{21}$ Previous studies have performed to evaluate the risk of LNM in cervical cancer. Several prognostic factors including FIGO stage, lymphatic permeation, and tumor histologic type have been demonstrated to be associated with LNM in cervical cancer. ${ }^{22}$ Our study was consistent with these published reports. Moreover, we found that GTV and the maximum diameter were more accurate than FIGO stage in predicting LNM or LVSI in this study.

As for the protocol of our study, we claimed the maximum diameter of tumor to assess the $\mathrm{N}$ stages and identify LVSI of cervical cancer for the first time. In the revised FIGO

TABLE 2. Univariate analysis of clinicopathological factors and gross tumor size of resectable cervical cancer correlated with LVSI

\begin{tabular}{|c|c|c|c|}
\hline \multirow[b]{2}{*}{ Variables } & \multicolumn{2}{|c|}{ LVSI } & \multirow[b]{2}{*}{$P$} \\
\hline & $\begin{array}{l}\text { Negative } \\
(n=177)\end{array}$ & $\begin{array}{l}\text { Positive } \\
(n=78)\end{array}$ & \\
\hline Age, year & & & 0.903 \\
\hline$<50$ & $107(67.7)$ & $51(32.3)$ & \\
\hline$\geq 50$ & $70(72.2)$ & $27(27.8)$ & \\
\hline Histology type & & & 0.352 \\
\hline Adenocarcinoma & $14(60.9)$ & $9(39.1)$ & \\
\hline $\begin{array}{l}\text { Squamous cell } \\
\text { carcinoma }\end{array}$ & $163(70.3)$ & $69(29.7)$ & \\
\hline Tumor differentiation & & & 0.014 \\
\hline Poorly differentiated & $95(66.0)$ & $49(34.0)$ & \\
\hline Moderately differentiated & $29(54.7)$ & $24(45.3)$ & \\
\hline Highly differentiated & $53(91.4)$ & $5(9.6)$ & \\
\hline FIGO category & & & $<0.0001$ \\
\hline IB1 & $55(82.1)$ & $12(17.9)$ & \\
\hline IB2 & $57(83.8)$ & $11(16.2)$ & \\
\hline IIA 1 & $35(62.5)$ & $21(37.5)$ & \\
\hline IIA2 & $10(45.5)$ & $12(54.5)$ & \\
\hline IIB & $20(47.6)$ & $22(52.4)$ & \\
\hline $\begin{array}{l}\text { Maximum diameter } \\
\text { of tumor, } \mathrm{cm}\end{array}$ & & & $<0.0001$ \\
\hline$<4$ & $162(72.6)$ & $61(27.4)$ & \\
\hline$\geq 4$ & $15(46.9)$ & $17(53.1)$ & \\
\hline GTV, $\mathrm{cm}^{3}$ & & & $<0.0001$ \\
\hline$<3.9$ & $98(77.2)$ & $29(22.8)$ & \\
\hline$\geq 3.9$ & $79(61.7)$ & $49(38.3)$ & \\
\hline LNM & & & $<0.0001$ \\
\hline Absent & $133(78.7)$ & $36(21.3)$ & \\
\hline Present & $44(51.2)$ & $42(48.8)$ & \\
\hline
\end{tabular}

Numbers in parentheses are the percentages. 

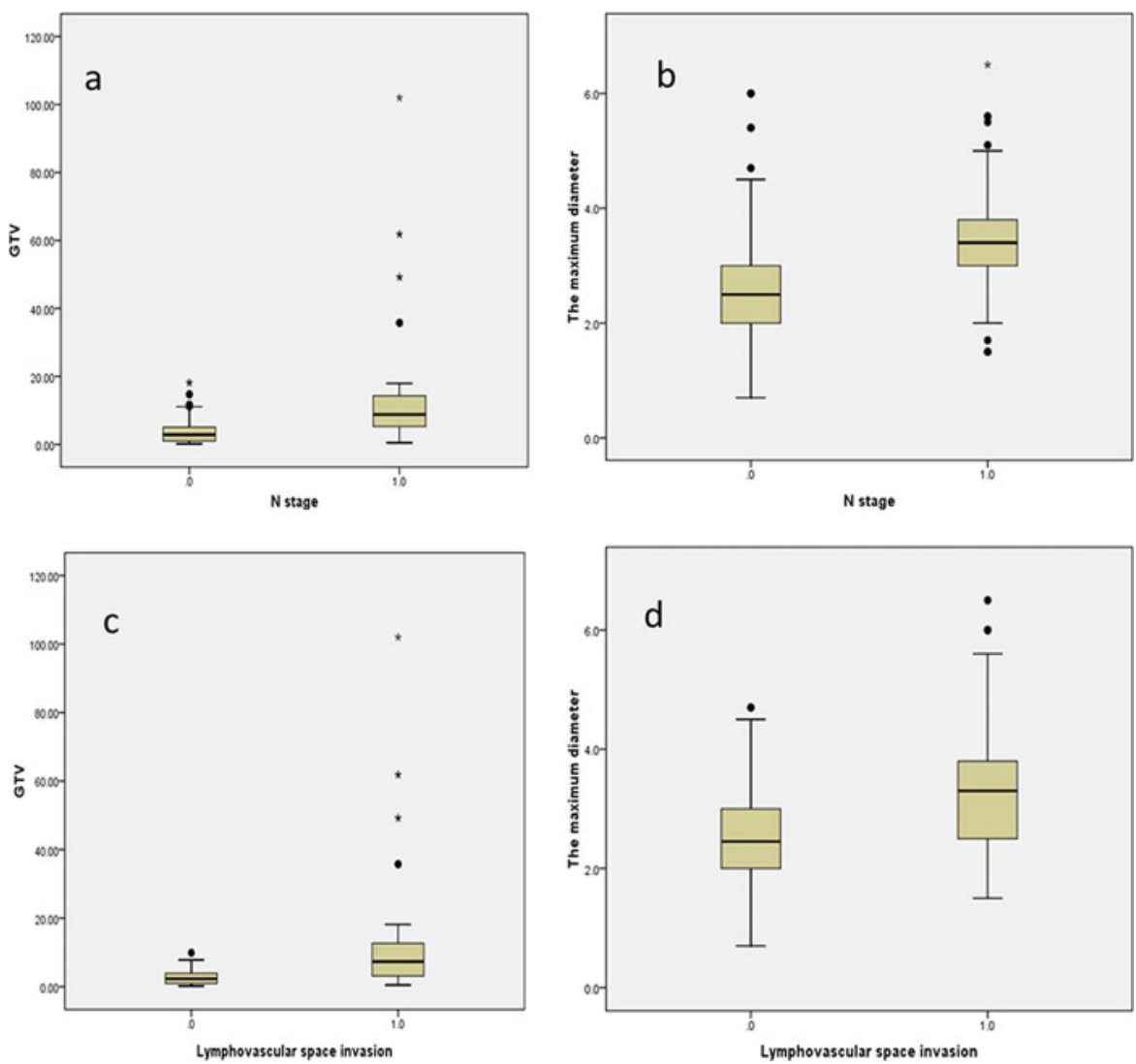

FIGURE 2. Box plots show the correlation between the GTV (in centimeters cube) and $N$ stage (A), the maximum diameter of tumor (in centimeters) and N stage (B), and the distributions of GTV (C) and the maximum diameter of tumor (D) stratified by LVSI.

staging for cervical cancer, the accurate division of tumor size (the maximum diameter with a cutoff of $4 \mathrm{~cm}$ ) has been used for staging. ${ }^{5}$ As suggested in the published article, tumor diameter was correlated with FIGO stage, LNM, and tumor recurrence rate. ${ }^{23,24}$ It is mostly reported that the larger the tumor diameter, the broader the surrounding invasion and the

TABLE 3. ROC analysis for tumor size of resectable cervical cancer for detecting LNM and LVSI

\begin{tabular}{|c|c|c|c|c|c|c|c|}
\hline Group & Cutoff Value & AUC & Sensitivity & Specificity & PPV & NPV & Accuracy \\
\hline \multicolumn{8}{|c|}{ Development cohort $(\mathrm{n}=255)$} \\
\hline \multicolumn{8}{|c|}{ N0 vs N1 } \\
\hline GTV & $5.17 \mathrm{~cm}^{3}$ & 0.813 & 0.756 & 0.760 & 0.613 & 0.859 & 0.757 \\
\hline Maximum diameter & $2.75 \mathrm{~cm}$ & 0.741 & 0.802 & 0.614 & 0.510 & 0.857 & 0.670 \\
\hline \multicolumn{8}{|l|}{$\operatorname{LVSI}(+)$ vs $(-)$} \\
\hline GTV & $6.41 \mathrm{~cm}^{3}$ & 0.806 & 0.602 & 0.934 & 0.601 & 0.934 & 0.760 \\
\hline Maximum diameter & $2.90 \mathrm{~cm}$ & 0.751 & 0.684 & 0.674 & 0.633 & 0.843 & 0.608 \\
\hline \multicolumn{8}{|l|}{ Validation cohort $(n=60)$} \\
\hline \multicolumn{8}{|l|}{ N0 vs N1 } \\
\hline GTV & $5.17 \mathrm{~cm}^{3}$ & 0.902 & 0.789 & 0.853 & 0.714 & 0.897 & 0.833 \\
\hline Maximum diameter & $2.75 \mathrm{~cm}$ & 0.825 & 0.842 & 0.610 & 0.531 & 0.928 & 0.716 \\
\hline \multicolumn{8}{|l|}{ LVSI $(+)$ vs $(-)$} \\
\hline GTV & $6.41 \mathrm{~cm}^{3}$ & 0.771 & 0.600 & 0.850 & 0.600 & 0.825 & 0.750 \\
\hline Maximum diameter & $2.90 \mathrm{~cm}$ & 0.748 & 0.750 & 0.625 & 0.500 & 0.833 & 0.748 \\
\hline
\end{tabular}

NPV, negative predictive value; PPV, positive predictive value. 

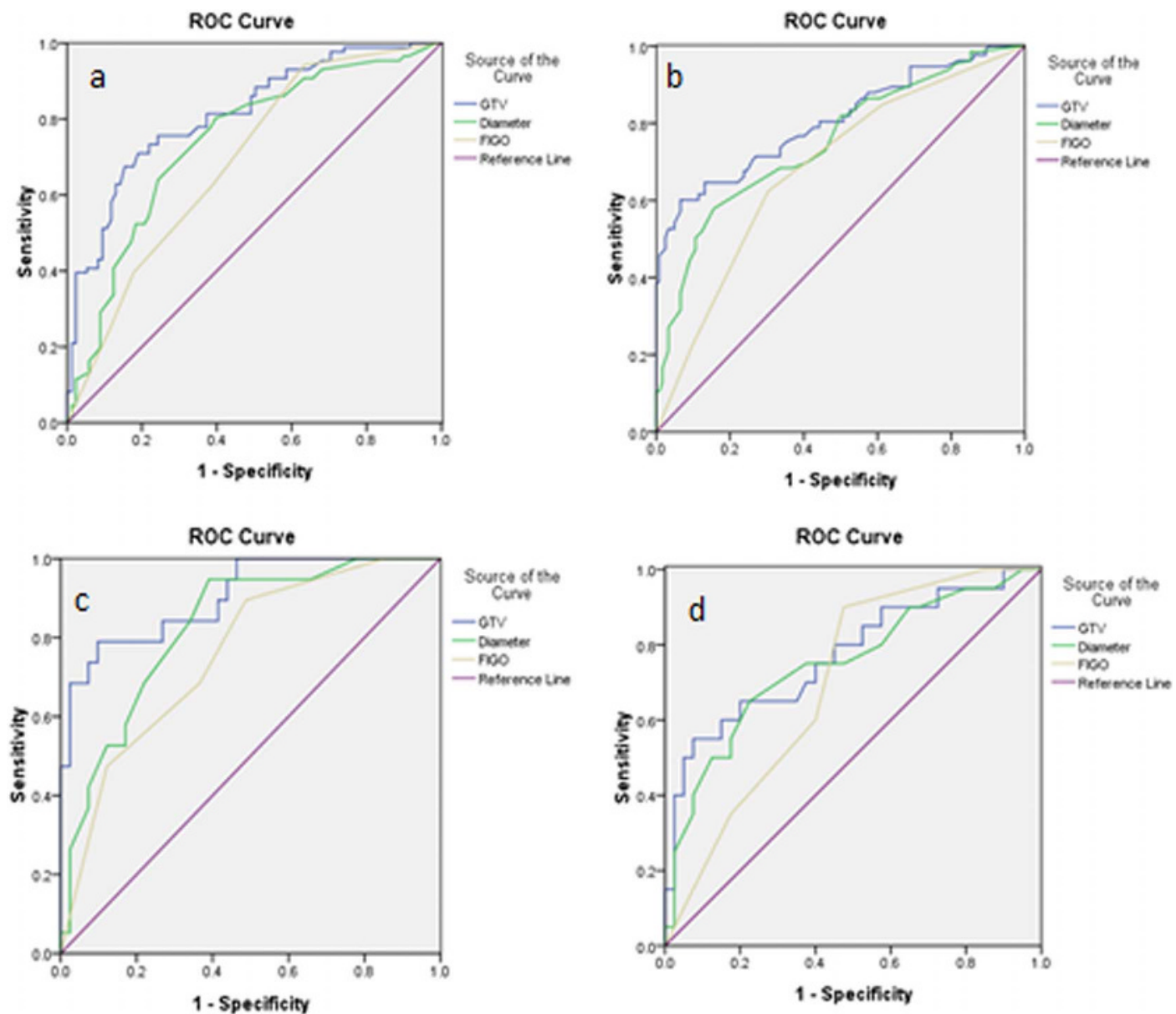

FIGURE 3. Receiver operating characteristic curves of GTV, the maximum diameter, and the FIGO classification of tumor for detecting LNM and LVSI in patients with of resectable cervical cancer in the development cohort (A, B) and in the validation cohort $(C, D)$, respectively. The ROC curve shows that GTV could be better for detecting LNM and LVSI.

greater risk of LNM.25 The maximum diameter of tumor at MRI could be used for identifying the presence of LNM and LVSI in resectable cervical cancer patients with cutoff value of 2.75 and 2.90 $\mathrm{cm}$, respectively. The previously reported studies confirmed that the tumor diameter of at least $25 \mathrm{~mm}$ on MRI could be an independent risk factor for parametrial invasion in cervical cancer with FIGO stage IB $1 .{ }^{26}$ Our study confirmed the previously mentioned results that the maximum diameter of resectable cervical cancer correlated with regional LNM and LVSI.

In this study, we found that GTV was not only correlated with regional LNM and LVSI, but also an independent risk factor to detect the occurrence of LNM and LVSI. These results suggest that GTV based on MRI could be a potential alternative technique for the preoperative identifying LVSI and distinguishing $\mathrm{N}$ stages of cervical cancer. The "objective" MRI compared with "subjective" visual assessment had decreased observer dependence and increased diagnostic performance in evaluating cervical cancer. ${ }^{27}$ The previous investigator showed that the similar index (metabolic tumor volume) also could predict the tumor relapse and the survival of patients with cervical cancer. ${ }^{28,29}$ Previous studies found that tumor volume of head-neck cancer and endometrial cancer on MRI was the most important independent factor associated with the tumor grade and overall survival. ${ }^{30,31}$ Our results showed that GTV could help identify the presence of LNM and LVSI with cutoff values of 5.17 and $6.41 \mathrm{~cm}^{3}$, respectively. The probably pathological mechanism could be that LVSI was mainly around the tumor. The larger the GTV, the deeper the surrounding invasion and there was more likely to involve LVSI and the more frequent the incidence of LNM. ${ }^{25,31}$ Compared with traditional FIGO classification, GTV of cervical cancer measured on MRI could be more accurate and objective for predicting the LNM. ${ }^{27}$ Owing to the subjectivity of the different gynecologic oncologists with different work experience, there may be different clinical FIGO stage for the same cervical cancer patients. Therefore, the noninvasive MRI assessment of LNM and the LVSI plays an important role in determining whether these patients should undergo extensive lymphadenectomies. 
In our study, there were also some limitations. First of all, this study only included the cervical cancer patients who received lymphadenectomy and the patients who did not undergo lymphadenectomy were excluded from this study. Our inclusion criteria might be provided in case selection bias. Despite this limitation, our study indicated that GTV and the maximum diameter of resectable cervical cancer at MRI could predict the presence of LNM and LVSI. Second, we have not routinely assessed imaging features including parametrial invasion or size of lymph nodes on the MRI in this article. However, many previous studies had demonstrated that MRI features could help differentiate the stage of cervical cancer, ${ }^{32}$ but with low sensitivity $(29 \%-86 \%)$ in detecting LNM for its incapability to detect micrometastases in normal-sized nodes. ${ }^{11,12}$ In our future study, we would like to compare the accuracy of tumor size or node size in predicting the $\mathrm{N}$ stage.

In conclusion, GTV and the maximum diameter of resectable cervical cancer at MRI demonstrated to be helpful in quantitatively predicting the presence of LNM and LVSI, which were more accurate than FIGO stage in predicting LNM or LVSI. We believe that preoperative assessment of the status of lymph nodes and LVSI is important in formulating individualized treatment plan for the individual cases.

\section{REFERENCES}

1. Torre LA, Bray F, Siegel RL, et al. Global cancer statistics, 2012. CA Cancer J Clin. 2015;65:87-108.

2. Haltia UM, Bützow R, Leminen A, et al. FIGO 1988 versus 2009 staging for endometrial carcinoma: a comparative study on prediction of survival and stage distribution according to histologic subtype. J Gynecol Oncol. 2014;25:30-35.

3. Koh WJ, Greer BE, Abu-Rustum NR, et al. Cervical cancer, version 2.2015. J Natl Compr Canc Netw. 2015;13:395-404.

4. Shirazi AS, Razi T, Cheraghi F, et al. Diagnostic accuracy of magnetic resonance imaging versus clinical staging in cervical cancer. Asian Pac J Cancer Prev. 2014;15:5729-5732.

5. Pecorelli S, Zigliani L, Odicino F. Revised FIGO staging for carcinoma of the cervix. Int J Gynaecol Obstet. 2009; 105:107-108.

6. Kim HS, Song YS. International Federation of Gynecology and Obstetrics (FIGO) staging system revised: what should be considered critically for gynecologic cancer? J Gynecol Oncol. 2009;20:135-136.

7. Twu NF, Ou YC, Liao CI, et al. Prognostic factors and adjuvant therapy on survival in early-stage cervical adenocarcinoma/ adenosquamous carcinoma after primary radical surgery: a Taiwanese Gynecologic Oncology Group (TGOG) study. Surg Oncol. 2016;25:229-235.

8. Epstein E, Testa A, Gaurilcikas A, et al. Early-stage cervical cancer: tumor delineation by magnetic resonance imaging and ultrasound-a European multicenter trial. Gynecol Oncol. 2013;128:449-453.

9. Pálsdóttir K, Fischerova D, Franchi D, et al. Preoperative prediction of lymph node metastasis and deep stromal invasion in women with invasive cervical cancer: prospective multicenter study using 2D and 3D ultrasound. Ultrasound Obstet Gynecol. 2015;45:470-475.

10. Balleyguier C, Sala E, Da Cunha T, et al. Staging of uterine cervical cancer with MRI: guidelines of the European Society of Urogenital Radiology. Eur Radiol. 2011;21:1102-1110.
11. Chung HH, Kang KW, Cho JY, et al. Role of magnetic resonance imaging and positron emission tomography/computed tomography in preoperative lymph node detection of uterine cervical cancer. Am J Obstet Gynecol. 2010;203: 156.e1-156.e5.

12. Liyanage SH, Roberts CA, Rockall AG. MRI and PET scans for primary staging and detection of cervical cancer recurrence. Womens Health (Lond). 2010;6:251-267.

13. Yang WT, Lam WW, Yu MY, et al. Comparison of dynamic helical CT and dynamic MR imaging in the evaluation of pelvic lymph nodes in cervical carcinoma. AJR Am J Roentgenol. 2000;175:759-766

14. Choi HJ, Ju W, Myung SK, et al. Diagnostic performance of computer tomography, magnetic resonance imaging, and positron emission tomography or positron emission tomography/computer tomography for detection of metastatic lymph nodes in patients with cervical cancer: meta-analysis. Cancer Sci. 2010;101:1471-1479.

15. Sironi S, Buda A, Picchio M, et al. Lymph node metastasis in patients with clinical early-stage cervical cancer: detection with integrated FDG PET/CT. Radiology. 2006;238: 272-279.

16. Mitchell DG, Snyder B, Coakley F, et al. Early invasive cervical cancer: MRI and CT predictors of lymphatic metastases in the ACRIN 6651/GOG 183 intergroup study. Gynecol Oncol. 2009;112:95-103.

17. Murakami I, Fujii T, Kameyama K, et al. Tumor volume and lymphovascular space invasion as a prognostic factor in early invasive adenocarcinoma of the cervix. J Gynecol Oncol. 2012;23:153-158.

18. Park JY, Kim DY, Kim JH, et al. Outcomes after radical hysterectomy according to tumor size divided by 2-cm interval in patients with early cervical cancer. Ann Oncol. 2011;22:59-67.

19. Mongula JE, Slangen BF, Lambregts DM, et al. Consecutive magnetic resonance imaging during brachytherapy for cervical carcinoma: predictive value of volume measurements with respect to persistent disease and prognosis. Radiat Oncol. 2015;10:252.

20. Mayr NA, Taoka T, Yuh WT, et al. Method and timing of tumor volume measurement for outcome prediction in cervical cancer using magnetic resonance imaging. Int J Radiat Oncol Biol Phys. 2002;52:14-22.

21. Kidd EA, Siegel BA, Dehdashti F, et al. Lymph node staging by positron emission tomography in cervical cancer: relationship to prognosis. J Clin Oncol. 2010;28:2108-2113.

22. Li D, Cai J, Kuang Y, et al. Surgical-pathologic risk factors of pelvic lymph node metastasis in stage Ib1-IIb cervical cancer. Acta Obstet Gynecol Scand. 2012;91:802-809.

23. Kesic V, Rodolakis A, Denschlag D, et al. Fertility preserving management in gynecologic cancer patients: the need for centralization. Int J Gynecol Cancer. 2010;20:1613-1619.

24. Yan M, Zhang YN, He JH, et al. Prognosis analysis of 83 cases of cervical adenosquamous carcinoma. Ai Zheng. 2008;27:956-961.

25. Baalbergen A, Ewing-Graham PC, Hop WC, et al. Prognostic factors in adenocarcinoma of the uterine cervix. Gynecol Oncol. 2004,92:262-267.

26. Yamazaki H, Todo Y, Okamoto K, et al. Pretreatment risk factors for parametrial involvement in FIGO stage IB1 cervical cancer. J Gynecol Oncol. 2015;26:255-261.

27. Mongula J, Slangen B, Lambregts D, et al. Predictive criteria for MRI-based evaluation of response both during and after 
radiotherapy for cervical cancer. $J$ Contemp Brachyther. 2016;8:181-188.

28. Yang Z, Xu W, Ma Y, et al. (18)F-FDG PET/CT can correct the clinical stages and predict pathological parameters before operation in cervical cancer. Eur J Radiol. 2016;85:877-884.

29. Kim BS, Kim IJ, Kim SJ, et al. The prognostic value of the metabolic tumor volume in FIGO stage IA to IIB cervical cancer for tumor recurrence: measured by F-18 FDG PET/CT. $\mathrm{Nucl}$ Med Mol Imaging. 2011;45:36-42.

30. Plataniotis GA, Theofanopoulou ME, Kalogera-Fountzila A, et al. Prognostic impact of tumor volumetry in patients with locally advanced head-and-neck carcinoma (non-nasopharyngeal) treated by radiotherapy alone or combined radiochemotherapy in a randomized trial. Int J Radiat Oncol Biol Phys. 2004;59:1018-1026.

31. Nougaret S, Reinhold C, Alsharif SS, et al. Endometrial cancer: combined MR volumetry and diffusion-weighted imaging for assessment of myometrial and lymphovascular invasion and tumor grade. Radiology. 2015;276:797-808.

32. Freeman SJ, Aly AM, Kataoka MY, et al. The revised FIGO staging system for uterine malignancies: implications for MR imaging. Radiographics. 2012;32:1805-1827. 